Grażyna Kwiecińska, Institute of Mathematics, University of Gdańsk, Wita Stwosza 57, 80-952 Gdańsk, Poland. e-mail: gkk@ksinet.univ.gda.p1

\title{
ON THE INTERMEDIATE VALUE PROPERTY OF MULTIVALUED FUNCTIONS
}

\begin{abstract}
The present paper deals with a certain property of multivalued functions which coincides with the Darboux property in the case of single valued real functions of real variable. It is well known that derivatives, and hence approximately continuous functions have the Darboux property. The results contained here are generalizations of these properties to the multivalued case.
\end{abstract}

\section{Preliminaries}

Let $X$ and $Y$ be two nonempty sets. A multivalued function $F: X \rightarrow Y$ is an mapping from $X$ to the nonempty subsets of $Y$; thus, for each $x \in X, F(x)$ is a nonempty set in $Y$.

For $F: X \rightarrow Y$ and $A \subset X$ and $B \subset Y$ we denote the image of $A$ and two counterimages of $B$ as follows:

$F(A)=\bigcup\{F(x): x \in A\}$,

$F^{+}(B)=\{x \in X: F(x) \subset B\}$, and $F^{-}(B)=\{x \in X: F(x) \cap B \neq \emptyset\}$.

Let us note that $F^{-}(B)=X \backslash F^{+}(Y \backslash B)$.

Let $(X, \mathcal{T}(X))$ and $(Y, \mathcal{T}(Y))$ be topological spaces. A multivalued function $F: X \rightarrow Y$ is called upper (resp. lower) semicontinuous at a point $x \in X$ if

$$
\begin{aligned}
& \forall G \in \mathcal{T}(Y)\left(F(x) \subset G \Rightarrow x \in \operatorname{Int} F^{+}(G)\right) \\
& \left(\text { resp. } \forall G \in \mathcal{T}(Y)\left(F(x) \cap G \neq \emptyset \Rightarrow x \in \operatorname{Int} F^{-}(G)\right)\right) .
\end{aligned}
$$

$F$ is called continuous at $x$ if it is simultaneously upper and lower semicontinuous at $x$.

Key Words: set-valued functions; continuity, Darboux property, differentiation of setvalued functions

Mathematical Reviews subject classification: 26A15, 26E25, 54C60, 26A24

Received by the editors December 22, 1999 
In case $(Y, d)$ is a metric space, we have more notation. We use $B(x, r)$ to denote an open ball and $B(A, r)=\bigcup\{B(x, r): x \in A\}$.

A multivalued function $F: X \rightarrow Y$ is called $h$-upper (resp. h-lower) semicontinuous at the point $x_{0} \in X$ if for each $\varepsilon>0$ there exists a neighborhood $U\left(x_{0}\right)$ of $x_{0}$ such that $F(x) \subset B\left(F\left(x_{0}\right), \varepsilon\right.$ ) (resp.) $F\left(x_{0}\right) \subset B(F(x), \varepsilon)$ ) for each $x \in U\left(x_{0}\right) . F$ is $h$-continuous at $x_{0}$ if it is simultaneously $h$-upper and $h$-lower semicontinuous at $x_{0}$.

It is known that if $F$ is upper semicontinuous at $x \in X$, then $F$ is $h$-upper semicontinuous at $x$, while if $F$ is $h$-lower semicontinuous at $x \in X$, then $F$ is lower semicontinuous at $x$ ([Lc, Th. 1.15 and 1.12]). If moreover the $F(x)$ are compact for $x \in X$, upper and $h$-upper semicontinuity are equivalent, as are lower and $h$-lower semicontinuity ([Lc, Th. 1.17 and 1.14]).

Let $\mathcal{P}(Y)$ denote the family of all subsets of $Y$ and let $\mathcal{P}_{0}(Y)=\mathcal{P}(Y) \backslash\{\emptyset\}$. We define the following families of sets:

$$
\begin{aligned}
\mathcal{C}(Y) & =\left\{A \in \mathcal{P}_{0}(Y): A \text { is closed }\right\} \\
\mathcal{C}_{b}(Y) & =\left\{A \in \mathcal{P}_{0}(Y): A \text { is closed and bounded }\right\} .
\end{aligned}
$$

For $A, B \in \mathcal{C}_{b}(Y)$ let $d_{H}(A, B)$ denote the Hausdorff metric of the sets $A$ and $B$. Then the set $\mathcal{C}_{b}(Y)$ with the Hausdorff metric becomes a metric space.

Theorem A. If $F: X \rightarrow Y$ has closed and bounded values, then $F$ is $h$ continuous iff $F$ is continuous (with respect to $d_{H}$ ) as a function from $X$ to $\mathcal{C}_{b}(Y)$.

Let $(Y,\|\cdot\|)$ be a real normed linear space and let $\mathbb{R}$ denote the set of real numbers. The symbol $\mathcal{C}_{o b}(Y)$ will denote the collection of all nonempty, closed, bounded and convex subsets of $Y$.

If $A \subset Y, B \subset Y$, and $\lambda \in \mathbb{R}$, let

$$
A+B=\{a+b: a \in A, b \in B\}, \lambda A=\{\lambda a: a \in A\}, A-B=A+(-1) B .
$$

We will write $A+x$ instead of $A+\{x\}$.

Theorem B. Let $A, B, C \in \mathcal{P}(Y)$.

(i) If $A$ is convex, $\alpha \geq 0$ and $\beta \geq 0$, then $(\alpha+\beta) A=\alpha A+\beta A$.

(ii) If $A$ and $B$ are closed and convex and $C$ is bounded, then $A+C=B+C$ implies $A=B$ (see [Rd, Lemma 2]).

(iii) If $A_{i}, B_{i} \in \mathcal{C}_{b}(Y)$ for $i=1,2$, then $d_{H}\left(A_{1}+A_{2}, B_{1}+B_{2}\right) \leq d_{H}\left(A_{1}, B_{1}\right)+$ $d_{H}\left(A_{2}, B_{2}\right)$. 
(iv) If $Y$ is reflexive and $A, B \in \mathcal{C}_{o b}(Y)$, then $A+B \in \mathcal{C}_{o b}(Y)$.

(v) If moreover $C \in \mathcal{C}_{o b}(Y)$, then $d_{H}(A, B)=d_{H}(A+C, B+C)$ (see [Rd, Th. 2]).

If $Y$ is complete, then $\left(\mathcal{C}_{b}(Y), d_{H}\right)$ is also complete (see [Kt, p. 314]). Therefore Price's inequality (see [Pc. (2.9), p. 4])

$$
d_{H}(\operatorname{co}(A), \operatorname{co}(B)) \leq d_{H}(A, B)
$$

where $\operatorname{co}(A)$ denotes the convex hull of $A$, implies the following.

Theorem C. A Cauchy sequence in $\mathcal{C}_{o b}(Y)$ must converge to an element of $\mathcal{C}_{o b}(Y)$.

Let $\mathcal{L}(\mathbb{R})$ denote the $\sigma$-field of Lebesgue measurable subsets of $\mathbb{R}$. Let $T \in \mathcal{L}(\mathbb{R})$ and let $(Y, \mathcal{T}(Y))$ be a topological space.

A multivalued function $F: T \rightarrow Y$ is called upper (resp. lower) measurable if $F^{+}(A)$ is measurable for any open (resp. closed) subset $A$ of $Y$.

The following assertion is known.

Theorem D. If $(Y, \mathcal{T}(Y))$ is perfect and $F$ is upper measurable, then $F$ is lower measurable (see [KŚ, Prop. 1 (i)]).

If $(Y, \mathcal{T}(Y))$ is perfectly normal and $F$ has compact values, then upper and lower measurability of $F$ are equivalent (see [KŚ, Prop. 1 (ii)]).

\section{The $\mathcal{D}_{*}$ Property of Continuous Multivalued Functions}

Let $(X, \mathcal{T}(X))$ and $(Y, \mathcal{T}(Y))$ be topological spaces. In [EL] the following definition of a Darboux property was given.

Definition 1. A multivalued function $F: X \rightarrow Y$ will be said to have the Darboux property (or $\mathcal{D}$ property) if for every connected set $C \subset X$, the image $F(C)$ is connected in $Y$.

Let $I \subset \mathbb{R}$ be an interval. For each $a, b \in \mathbb{R}$ we will use $a \wedge b$ and $a \vee b$ to denote the minimum and maximum, respectively, of $a$ and $b$. In [CK] the following definition was introduced.

Definition 2. A multivalued function $F: I \rightarrow \mathbb{R}$ will be said to have intermediate value property (or $\mathcal{D}_{*}$ property) if for each pair of distinct points $x_{1}, x_{2} \in I$ and each $y_{1} \in F\left(x_{1}\right)$ there exists $y_{2} \in F\left(x_{2}\right)$ such that $\left(y_{1} \wedge y_{2}, y_{1} \vee\right.$ $\left.y_{2}\right) \subset F\left(\left(x_{1} \wedge x_{2}, x_{1} \vee x_{2}\right)\right)$. 
Note each of the properties $\mathcal{D}$ and $\mathcal{D}_{*}$ is equivalent to the usual Darboux property when $F(x)=\{f(x)\}$, where $f: I \rightarrow \mathbb{R}$ is a function.

The following examples show that they are not equivalent in general.

Example 1. The multivalued function $F_{1}: \mathbb{R} \rightarrow \mathbb{R}$ defined by the formula

$$
F_{1}(x)= \begin{cases}{[0,2],} & \text { if } x=0, \\ {[0,1],} & \text { if } x \neq 0,\end{cases}
$$

has the $\mathcal{D}$ property, but not the $\mathcal{D}_{*}$ property.

Example 2. Let $F_{2}(x)=[0,1] \cup[2,3]$ for each $x \in \mathbb{R}$. Then $F_{2}$ has the $\mathcal{D}_{*}$ property and does not have the $\mathcal{D}$ property $([\mathrm{CK}])$.

Note that $F_{2}$ is continuous. Therefore, a continuous multivalued function (with closed values) does not necessarily have the $\mathcal{D}$ property, but it does have the $\mathcal{D}_{*}$ property .

Theorem 1. If a multivalued function $F: I \rightarrow \mathbb{R}$ with closed values is continuous, then it has the intermediate value property.

Proof. Assume the contrary. Then

there exist two distinct points $x_{1}, x_{2} \in I$ (say $x_{1}<x_{2}$ ) and a point $y_{1} \in F\left(x_{1}\right)$ such that for any $y_{2} \in F\left(x_{2}\right)$ a number $\alpha$ exists such that

$\alpha \in\left(y_{1} \wedge y_{2}, y_{1} \vee y_{2}\right) \backslash F\left(\left(x_{1}, x_{2}\right)\right)$.

Obviously $y_{1} \notin F\left(x_{2}\right)$ since otherwise taking $y_{2}=y_{1}$ contradicts (1). Let

$$
B_{1}=\left\{y \in F\left(x_{2}\right): y<y_{1}\right\} \text { and } B_{2}=\left\{y \in F\left(x_{2}\right): y>y_{1}\right\} .
$$

Since $F\left(x_{2}\right) \neq \emptyset$, at least one of the sets $B_{1}$ and $B_{2}$ is nonempty. Assume that $B_{1} \neq \emptyset$ and $B_{2} \neq \emptyset$, and let $y^{\prime}=\sup B_{1}, y^{\prime \prime}=\inf B_{2}$. Since $F\left(x_{2}\right)$ is a closed set, $y^{\prime}, y^{\prime \prime} \in F\left(x_{2}\right)$ and $y^{\prime}<y_{1}<y^{\prime \prime}$, and since $y_{1} \in F\left(x_{1}\right)$, by condition (1) there exist numbers $\alpha^{\prime}$ and $\alpha^{\prime \prime}$ such that $\alpha^{\prime} \in\left(y^{\prime}, y_{1}\right), \alpha^{\prime \prime} \in\left(y_{1}, y^{\prime \prime}\right)$ and $\alpha^{\prime}, \alpha^{\prime \prime} \notin F\left(\left(x_{1}, x_{2}\right)\right)$. This implies that

$$
F^{-}\left(\left(\alpha^{\prime}, \alpha^{\prime \prime}\right)\right) \cap\left(x_{1}, x_{2}\right)=F^{-}\left(\left[\alpha^{\prime}, \alpha^{\prime \prime}\right]\right) \cap\left(x_{1}, x_{2}\right) .
$$

Moreover $y_{1} \in\left(\alpha^{\prime}, \alpha^{\prime \prime}\right)$ and $y_{1} \in F\left(x_{1}\right)$. Hence $x_{1} \in F^{-}\left(\left(\alpha^{\prime}, \alpha^{\prime \prime}\right)\right)$. By the choice of $\alpha^{\prime}$ and $\alpha^{\prime \prime}, F\left(x_{2}\right) \subset\left(-\infty, \alpha^{\prime}\right) \cup\left(\alpha^{\prime \prime},+\infty\right)$. Hence $x_{2} \notin F^{-}\left(\left[\alpha^{\prime}, \alpha^{\prime \prime}\right]\right)$. We conclude that

$$
F^{-}\left(\left(\alpha^{\prime}, \alpha^{\prime \prime}\right)\right) \cap\left[x_{1}, x_{2}\right]=F^{-}\left(\left[\alpha^{\prime}, \alpha^{\prime \prime}\right]\right) \cap\left[x_{1}, x_{2}\right] .
$$


Let $A=F^{-}\left(\left(\alpha^{\prime}, \alpha^{\prime \prime}\right)\right) \cap\left[x_{1}, x_{2}\right]$. By the continuity of $F$ and (2) the set $A$ is both open and closed in $\left[x_{1}, x_{2}\right]$. This is a contradiction since $x_{1} \in A$ and $x_{2} \notin A$.

Suppose now that $B_{1}=\emptyset$ and $B_{2} \neq \emptyset$. Taking

$$
A=F^{-}\left(\left(-\infty, \alpha^{\prime \prime}\right)\right) \cap\left[x_{1}, x_{2}\right],
$$

where $\alpha^{\prime \prime} \in\left(y_{1}, y^{\prime \prime}\right) \backslash F\left(\left(x_{1}, x_{2}\right)\right), y^{\prime \prime}=\inf B_{2}$, we reach a contradiction just as before. In the case $B_{1} \neq \emptyset$ and $B_{2}=\emptyset$ consider instead

$$
A=F^{-}\left(\left(\alpha^{\prime},+\infty\right)\right) \cap\left[x_{1}, x_{2}\right],
$$

where $\alpha^{\prime} \in\left(y^{\prime}, y_{1}\right) \backslash F\left(\left(x_{1}, x_{2}\right)\right), y^{\prime}=\sup B_{1}$. In any case we get a contradiction, hence the proof is complete.

Remark 1. The assumption that the multivalued function have closed values is important. In order to illustrate this, let us consider the multivalued function $F: \mathbb{R} \rightarrow \mathbb{R}$ defined by the formula

$$
F(x)= \begin{cases}\left\{y: y=\frac{1}{k}, k \in \mathbb{Z} \backslash\{0\}\right\}, & \text { if } x \in(0,1), \\ \left\{y: y=0 \vee y=\frac{1}{k}, k \in \mathbb{Z} \backslash\{0\}\right\}, & \text { if } x \notin(0,1),\end{cases}
$$

where $\mathbb{Z}$ is the set of integers. Then $F$ is continuous but does not have the $\mathcal{D}_{*}$ property .

\section{The $\mathcal{D}_{*}$ Property of the Derivative of a Multivalued Function}

Let $(Y,\|\cdot\|)$ be a reflexive real normed linear space with the metric $d$ determined by the norm in $Y$.

We define a difference $A \ominus B$ of the sets $A, B \in \mathcal{C}_{o b}(Y)$ as follows:

We will say the difference $A \ominus B$ is defined if there exists a set $C \in \mathcal{C}_{o b}(Y)$ such that either $A=B+C$ or $B=A-C$, and we define $A \ominus B$ to be $C$.

Example 3. Let $A=\alpha P$ and $B=\beta P$, where $P \in \mathcal{C}_{o b}(Y), \alpha \geq 0$ and $\beta \geq 0$. Put $C=(\alpha-\beta) P$. Then by Theorem B (i) $A=B+C$ or $B=A-C$ depending on whether $\alpha \geq \beta$ or $\alpha<\beta$. Therefore $\alpha P \ominus \beta P$ exists and is equal to $(\alpha-\beta) P$.

Example 4. Let

$$
\begin{aligned}
& A=\left\{(x, y) \in \mathbb{R}^{2}: 0 \leq x \leq 1, \quad 0 \leq y \leq 1-x\right\} \\
& B=\left\{(x, y) \in \mathbb{R}^{2}: 0 \leq x \leq 1, \quad 0 \leq y \leq \frac{1}{2}(1-x)\right\}
\end{aligned}
$$


Then $A \ominus B$ does not exist. Indeed, suppose that there exists $C \in \mathcal{C}_{o b}\left(\mathbb{R}^{2}\right)$ such that $A=B+C$. Since $(0,1) \in A$, there exist $(a, b) \in B$ and $(c, d) \in C$ such that $(0,1)=(a+c, b+d)$, where $a \geq 0$. Then $c=-a$ and $d=1-b$. On the other hand $(0,0) \in B$. Therefore $(0,0)+(c, d)=(-a, 1-b) \in A$ and $-a \geq 0$. Hence $a=0$. Since $(c, d)=(0,1-b) \in C$ and $(1,0) \in B$, we have $(1,0)+(0,1-b) \in A$ and $b=1$. Therefore we have $(a, b)=(0,1) \notin B$, which is a contradiction. Now let us suppose that there exists $C \in \mathcal{C}_{o b}\left(\mathbb{R}^{2}\right)$ such that $B=A-C$. Let $z \in C$. We observe that for every $x \in A, x-z \in A-C=B$. Hence we have $A-z \subset B$; i.e., some translation of $A$ is contained in $B$, which is of course not possible.

Theorem 2. Suppose $A \in \mathcal{C}_{o b}(Y)$ and $B \in \mathcal{C}_{o b}(Y)$.

(a) $\exists C \in \mathcal{C}_{o b}(Y) A=B+C \Longleftrightarrow \forall a \in \operatorname{Fr}(A) \exists y \in Y a \in B+y \subset A$.

(b) $\exists C \in \mathcal{C}_{o b}(Y) B=A-C \Longleftrightarrow \forall b \in \operatorname{Fr}(B) \exists y \in Y b \in A+y \subset B$,

where $\operatorname{Fr}(P)$ denotes the boundary of $P \subset Y$.

Proof. To prove (a), suppose the existence of $C \in \mathcal{C}_{o b}(Y)$ such that $A=$ $B+C$. If $a \in A$ (in particular $a \in \operatorname{Fr} A$ ), then $a \in B+C$. Therefore there are $b \in B$ and $c \in C$ such that $a=b+c$. If $z \in B$, then $z+c \in B+C=A$. Consequently $B+c \subset A$. Moreover $a=b+c \in B+c$. This proves that for $a \in \operatorname{Fr}(A)$ there is $y \in Y$ with $a \in B+y \subset A$.

Now let us suppose that for each $a \in \operatorname{Fr}(A)$ there exists $y \in Y$ such that $a \in B+y \subset A$. Let $C=\{x: B+x \subset A\}$. Then $C$ is closed and bounded. We will show that $C$ is convex. Let $c, c^{\prime} \in C$. Then $B+c \subset A$ and $B+c^{\prime} \subset A$. Let $\lambda \in[0,1]$. We obtain

$$
(1-\lambda)(B+c)+\lambda\left(B+c^{\prime}\right) \subset A .
$$

Furthermore

$$
(1-\lambda)(B+c)+\lambda\left(B+c^{\prime}\right)=B+(1-\lambda) c+\lambda c^{\prime} .
$$

We conclude from (3) and (4) that $B+(1-\lambda) c+\lambda c^{\prime} \subset A$. Hence that $z=(1-\lambda) c+\lambda c^{\prime} \in C$, and finally that $C$ is convex. Since $B+C \subset A$, we need to prove that $A \subset B+C$. Let $x \in A$. Since $A$ is convex, there exist $a, a^{\prime} \in \operatorname{Fr} A$ and $\lambda \in[0,1]$ such that $x=(1-\lambda) a+\lambda a^{\prime}$. Then by hypothesis there exist $y, y^{\prime} \in Y$ such that $a \in B+y \subset A$ and $a^{\prime} \in B+y^{\prime} \subset A$. Thus there exist $b, b^{\prime} \in B$ such that $a=b+y$ and $a^{\prime}=b^{\prime}+y^{\prime}$ and $x=(1-\lambda) a+\lambda a^{\prime}=$ $b^{\prime \prime}+(1-\lambda) y+\lambda y^{\prime}$, where $b^{\prime \prime}=(1-\lambda) b+\lambda b^{\prime}$. Thus $x \in B+(1-\lambda) y+\lambda y^{\prime}$. Since $y, y^{\prime} \in C$ and $C$ is convex, $u=(1-\lambda) y+\lambda y^{\prime} \in C$. Therefore $x \in B+C$, which finishes the proof of (a). 
To prove (b) we apply similar arguments, with $\{x: B+x \subset A\}$ replaced by $\{x: A-x \subset B\}$ in the second part of the proof.

Remark 2. We can replace Fr in Theorem 2 by the set of extreme points by appealing to the Krein-Milman theorem.

To see this, let us suppose that for all $a \in \operatorname{ex}(A)(\operatorname{ex}(A)$ means the set of extreme points of $A$ ) there exists $y \in Y$ with $a \in B+y \subset A$. The set $\operatorname{ex}(A)$ is nonempty since $A$ is compact. Let $C$ be as in the proof of Theorem 2. It suffices to show that $A \subset B+C$. For each $a \in \operatorname{ex}(A)$ there is $y \in C$ with $a \in B+y$ by hypothesis. Therefore $\operatorname{ex}(A) \subset B+C$. Then $\overline{c o}(\operatorname{ex}(A)) \subset B+C$ since $B+C$ is closed and convex, and finally $A \subset B+C$ by the Krein-Milman theorem.

It is easy to see that

If $B \in \mathcal{C}_{o b}(Y)$ and $y \in Y$, then $B+y \ominus B=\{y\}$. In particular $A \ominus A=\{0\}$.

If $A \ominus B$ exists, then $d_{H}(A, B)=\|A \ominus B\|$,

where $\|C\|=d_{H}(C,\{0\})$ for $C \subset Y$.

If $Y=\mathbb{R}$ and $A=[a, x]$ and $B=[b, y]$, then $A \ominus B$ exists and

$A \ominus B=[(a-b) \wedge(x-y),(a-b) \vee(x-y)]$.

Now we can present a definition of derivative of a multivalued function.

Definition 3. A multivalued function $F: I \rightarrow Y$ is said to be differentiable at $x_{0} \in I$ if there exists a set $D F\left(x_{0}\right) \in \mathcal{C}_{o b}(Y)$ such that the limit (with respect to the Hausdorff metric) $\lim _{x \rightarrow x_{0}} \frac{F(x) \ominus F\left(x_{0}\right)}{x-x_{0}}$ exists and is equal to $D F\left(x_{0}\right)$.

Of course, implicit in the definition of $D F\left(x_{0}\right)$ is the existence of the differences $F(x) \ominus F\left(x_{0}\right)$.

The set $D F\left(x_{0}\right)$ will be called the derivative of $F$ at $x_{0} . F$ will be called differentiable if it is differentiable at every point $x \in I$.

A multivalued function $G: I \rightarrow Y$ will be called a derivative if there exists a differentiable multivalued function $F: I \rightarrow Y$ with $G(x)=D F(x)$ for $x \in I$.

Example 5. Let $S$ be the closed unit ball in $\mathbb{R}^{2}$, and consider a multivalued function $F:(0,2 \pi) \rightarrow \mathbb{R}^{2}$ defined by $F(\alpha)=(2+\sin \alpha) S$. Then $F$ is differentiable and $D F(\alpha)=(\cos \alpha) S$ for each $\alpha \in(0,2 \pi)$.

Example 6. The multivalued function $F:[0,1] \rightarrow \mathbb{R}^{2}$ defined by the formula

$$
F(\alpha)=\left\{(x, y) \in \mathbb{R}^{2}: 0 \leq x \leq 1,0 \leq y \leq \alpha-\alpha x\right\}
$$

is not differentiable, since the required differences do not exist. 
Theorem 3. If a multivalued function $F: I \rightarrow Y$ with closed, bounded and convex values is differentiable at $x_{0} \in I$, then it is $h$-continuous at this point.

Proof. Suppose $F$ is differentiable at $x_{0}$. Let $x \neq x_{0}$. By the differentiability of $F$ at $x_{0}$, there exists a set $D F\left(x_{0}\right) \in \mathcal{C}_{o b}(Y)$ such that

$$
\lim _{x \rightarrow x_{0}} d_{H}\left(\frac{F(x) \ominus F\left(x_{0}\right)}{x-x_{0}}, D F\left(x_{0}\right)\right)=0 .
$$

Then (see (5))

$$
\begin{aligned}
d_{H}\left(F(x), F\left(x_{0}\right)\right) & =\left\|F(x) \ominus F\left(x_{0}\right)\right\|=\left\|\frac{F(x) \ominus F\left(x_{0}\right)}{x-x_{0}}\right\|\left|x-x_{0}\right| \\
& \leq\left(d_{H}\left(\frac{F(x) \ominus F\left(x_{0}\right)}{x-x_{0}}, D F\left(x_{0}\right)\right)+\left\|D F\left(x_{0}\right)\right\|\right)\left|x-x_{0}\right| .
\end{aligned}
$$

Since the set $D F\left(x_{0}\right)$ is bounded, (6) and (7) shows that $D_{H}\left(F(x), F\left(x_{0}\right)\right)$ converges to zero as $x$ tends to $x_{0}$. Hence, $F$ is $h$-continuous at $x_{0}$, by Theorem A.

Now we deal with the case when $Y=\mathbb{R}$. Let $F: I \rightarrow \mathbb{R}$ be a multivalued function with compact and convex values. Then

$$
F(x)=[i(x), s(x)],
$$

where $i(x)=\inf _{x \in I} F(x)$ and $s(x)=\sup _{x \in I} F(x)$.

If $F: I \rightarrow \mathbb{R}$ is differentiable at $x_{0} \in I$, then by Theorem 3

$F$ is $h$-continuous at $x_{0}$ and consequently the functions $i$ and $s$ are continuous at $x_{0}$.

It should be noted that in this case $F(x) \ominus F\left(x_{0}\right)$ exists for $x \in I$ and

$$
\frac{F(x) \ominus F\left(x_{0}\right)}{x-x_{0}}= \begin{cases}{\left[\frac{i(x)-i\left(x_{0}\right)}{x-x_{0}}, \frac{s(x)-s\left(x_{0}\right)}{x-x_{0}}\right],} & \text { if } \delta F(x) \geq \delta F\left(x_{0}\right), x>x_{0}, \\ {\left[\frac{s(x)-s\left(x_{0}\right)}{x-x_{0}}, \frac{i(x)-i\left(x_{0}\right)}{x-x_{0}}\right],} & \text { if } \delta F(x) \geq \delta F\left(x_{0}\right), x<x_{0}, \\ {\left[\frac{s(x)-s\left(x_{0}\right)}{x-x_{0}}, \frac{i(x)-i\left(x_{0}\right)}{x-x_{0}}\right],} & \text { if } \delta F(x) \leq \delta F\left(x_{0}\right), x>x_{0}, \\ {\left[\frac{i(x)-i\left(x_{0}\right)}{x-x_{0}}, \frac{s(x)-s\left(x_{0}\right)}{x-x_{0}}\right],} & \text { if } \delta F(x) \leq F\left(x_{0}\right), x<x_{0},\end{cases}
$$

where $\delta A$ denotes diameter of $A$.

The following can be easily verified. 
Theorem 4. If the functions $i: I \rightarrow \mathbb{R}$ and $s: I \rightarrow \mathbb{R}$ are differentiable at $x_{0} \in I$, then $F$ is differentiable at $x_{0}$ and

$$
D F\left(x_{0}\right)= \begin{cases}{\left[i^{\prime}\left(x_{0}\right), s^{\prime}\left(x_{0}\right)\right],} & \text { if } i^{\prime}\left(x_{0}\right) \leq s^{\prime}\left(x_{0}\right), \\ {\left[s^{\prime}\left(x_{0}\right), i^{\prime}\left(x_{0}\right)\right],} & \text { if } i^{\prime}\left(x_{0}\right)>s^{\prime}\left(x_{0}\right) .\end{cases}
$$

However, in general, differentiability of $F$ does not imply differentiability of the functions $i$ or $s$ as the following example shows.

$$
F(x)= \begin{cases}{[0, x],} & \text { if } x \leq 0, \\ {[x, 0],} & \text { if } x<0 .\end{cases}
$$

Let us suppose that $F$ is differentiable at $x_{0} \in I$. According to Definition 3, there is a set $D F\left(x_{0}\right) \in \mathcal{C}_{o b}(R)$ such that

$$
\lim _{x \rightarrow x_{0}} \frac{F(x) \ominus F\left(x_{0}\right)}{x-x_{0}}=D F\left(x_{0}\right) .
$$

This condition can be reinterpreted in terms of Dini derivatives of functions $i$ and $s$.

Let $f: I \rightarrow \mathbb{R}$ be a function and $x \in I$. We will use $f_{-}^{\prime}(x)$ and $f_{+}^{\prime}(x)$ to denote the left-side and right-side derivatives of $f$ at $x, D_{-} f(x), D^{-} f(x)$, $D_{+} f(x)$ and $D^{+} f(x)$ to denote the left and right lower and upper Dini derivatives of $f$ at $x$. Further, given $x, y \in I, x \neq y$, we define $Q f(x, y)$ by

$$
Q f(x, y)=\frac{f(x)-f(y)}{x-y} .
$$

Following Garg (see [G1] or [G2]), $f$ will be called lower derivable at $x$ if $D^{-} f(x) \leq D_{+} f(x)$ and if so, then the interval $\left[D^{-} f(x), D_{+} f(x)\right]$ will be called the lower derivative of $f$ at $x$ and denoted by $L f^{\prime}(x)$.

Similarly, $f$ is called upper derivable at $x$ if $D^{+} f(x) \leq D_{-} f(x)$ and if so, then $\left[D^{+} f(x), D_{-} f(x)\right]$ is called the upper derivative of $f$ at $x$ and denoted by $U f^{\prime}(x)$.

We will further call $f$ semiderivable at $x$ if it is either lower or upper derivable at $x$, and then its lower or upper derivative will be called the semiderivative of $f$ at $x$ and denoted by $S f^{\prime}(x)$.

If $D_{+} f(x) \leq D^{-} f(x)$, then $\left[D_{+} f(x), D^{-} f(x)\right]$ will be called the lower median of $f$ at $x$; and when $D_{-} f(x) \leq D^{+} f(x)$, the interval $\left[D_{-} f(x), D^{+} f(x)\right]$ will be called the upper median of $f$ at $x$. We will use $\underline{M} f(x)$ and $\bar{M} f(x)$ to denote the lower and upper median, respectively, of $f$ at $x$.

The following theorems will be useful. (See [G1, Th.5.1] or [G2, Th. 8.1.2] for Theorem E and [G1, Th. 9.3] or [G2, Th. 10.4.1] for Theorem F.) 
Theorem E. If a function $f:\left[x_{1}, x_{2}\right] \rightarrow \mathbb{R}$ is continuous, then there is a point $x \in\left(x_{1}, x_{2}\right)$ such that $f$ is semiderivable at $x$ and $Q f\left(x_{1}, x_{2}\right) \in S f^{\prime}(x)$.

Theorem F. Suppose $f: I \rightarrow \mathbb{R}$ is continuous. Then for each connected set $C \subset \mathbb{R}$ the set $\bigcup\left\{S f^{\prime}(x): x \in C \cap \Delta_{S}(f)\right\}$ is connected, where $\Delta_{S}(f)$ denotes the set of points in I where $f$ is semiderivable.

Let us suppose that $D F\left(x_{0}\right)=[a, b], a, b \in \mathbb{R}, a \leq b$. Then (10) and (11) force $a$ and $b$ to be the only limit points of $Q i\left(x, x_{0}\right)$ and $Q s\left(x, x_{0}\right)$.

If $a=b$, then the four Dini derivatives of $i$ and $s$ at $x_{0}$ are equal, and hence the functions $i$ and $s$ are differentiable at $x_{0}$ with $i^{\prime}\left(x_{0}\right)=s^{\prime}\left(x_{0}\right)$.

If $a<b$, then $i$ and $s$ may or may not be differentiable at $x_{0}$, but they do have a semiderivative at $x_{0}$ or a lower or upper median at $x_{0}$. Let us consider four basically different cases.

Case (i): There exists $h>0$ such that $\delta F(x) \geq \delta F\left(x_{0}\right)$ for each point $x \in\left(x_{0}, x_{0}+h\right)$, and $\delta F(x) \leq \delta F\left(x_{0}\right)$ for each $x \in\left(x_{0}-h, x_{0}\right)$. Then (11) holds iff $D_{+} i\left(x_{0}\right)=D^{+} i\left(x_{0}\right)=a, D_{+} s\left(x_{0}\right)=D^{+} s\left(x_{0}\right)=b$, and $D_{-} i\left(x_{0}\right)=$ $D^{-} i\left(x_{0}\right)=a, D_{-} s\left(x_{0}\right)=D^{-} s\left(x_{0}\right)=b$. Thus $i$ and $s$ are differentiable at $x_{0}$ and $D F\left(x_{0}\right)=\left[i^{\prime}\left(x_{0}\right), s^{\prime}\left(x_{0}\right)\right]$. Of course, $L i^{\prime}\left(x_{0}\right)=U i^{\prime}\left(x_{0}\right)=a$ and $U s^{\prime}\left(x_{0}\right)=L s^{\prime}\left(x_{0}\right)=b$.

Case (ii): There exists $h>0$ such that $\delta F(x) \geq \delta F\left(x_{0}\right)$ for each point $x \in\left(x_{0}, x_{0}+h\right)$, and $\delta F(x) \geq \delta F\left(x_{0}\right)$ for each $x \in\left(x_{0}-h, x_{0}\right)$. Then (11) holds iff $D_{+} i\left(x_{0}\right)=D^{+} i\left(x_{0}\right)=a, D_{+} s\left(x_{0}\right)=D^{+} s\left(x_{0}\right)=b$, and $D_{-} s\left(x_{0}\right)=$ $D^{-} s\left(x_{0}\right)=a, D_{-} i\left(x_{0}\right)=D^{-} i\left(x_{0}\right)=b$. Thus $i$ is upper derivable at $x_{0}, s$ is lower derivable at $x_{0}$, and $U i^{\prime}\left(x_{0}\right)=[a, b]=L s^{\prime}\left(x_{0}\right)=\left[i_{+}^{\prime}\left(x_{0}\right), s_{+}^{\prime}\left(x_{0}\right)\right]=$ $\left[s_{-}^{\prime}\left(x_{0}\right), i_{-}^{\prime}\left(x_{0}\right)\right]=D F\left(x_{0}\right)$.

Case (iii): There exists $h>0$ such that $\delta F(x) \geq \delta F\left(x_{0}\right)$ for each $x \in$ $\left(x_{0}, x_{0}+h\right)$ but for each $h>0$ there exists $x \in\left(x_{0}-h, x_{0}\right)$ such that $\delta F(x) \geq$ $\delta F\left(x_{0}\right)$ and there exists $x^{\prime} \in\left(x_{0}-h, x_{0}\right)$ such that $\delta F\left(x^{\prime}\right)<\delta F\left(x_{0}\right)$. Then (11) holds iff $D_{+} i\left(x_{0}\right)=D^{+} i\left(x_{0}\right)=a$ and $D_{+} s\left(x_{0}\right)=D^{+} s\left(x_{0}\right)=b, D_{-} s\left(x_{0}\right)=$ $a$ and $D^{-} s\left(x_{0}\right)=b, D_{-} i\left(x_{0}\right)=a$ and $D^{-} i\left(x_{0}\right)=b$. Thus $U i^{\prime}\left(x_{0}\right)=a$, $L s^{\prime}\left(x_{0}\right)=b$, and $\underline{M} i\left(x_{0}\right)=\bar{M} s\left(x_{0}\right)=\left[i_{+}^{\prime}\left(x_{0}\right), s_{+}^{\prime}\left(x_{0}\right)\right]=D F\left(x_{0}\right)$.

Case (iv): For each $h>0$ there exists $x \in\left(x_{0}, x_{0}+h\right)$ such that $\delta F(x) \geq$ $\delta F\left(x_{0}\right)$ and there exists $x^{\prime} \in\left(x_{0}, x_{0}+h\right)$ such that $\delta F\left(x^{\prime}\right)<\delta F\left(x_{0}\right)$, and for each $h>0$ there exists $x \in\left(x_{0}-h, x_{0}\right)$ such that $\delta F(x) \geq \delta F\left(x_{0}\right)$ and there exists $x^{\prime} \in\left(x_{0}-h, x_{0}\right)$ such that $\delta F\left(x^{\prime}\right)<\delta F\left(x_{0}\right)$. Then (11) holds iff $D_{+} i\left(x_{0}\right)=a$ and $D^{+} i\left(x_{0}\right)=b, D_{+} s\left(x_{0}\right)=a$ and $D^{+} s\left(x_{0}\right)=b, D_{-} i\left(x_{0}\right)=a$ and $D^{-} i\left(x_{0}\right)=b, D_{-} s\left(x_{0}\right)=a$ and $D^{-} s\left(x_{0}\right)=b$. Thus neither $i$ nor $s$ is semiderivable at $x_{0}$, and $\underline{M} i\left(x_{0}\right)=\bar{M} i\left(x_{0}\right)=\underline{M} s\left(x_{0}\right)=\bar{M} s\left(x_{0}\right)=D F\left(x_{0}\right)$.

We turn our attention to the well-known result on ordinary derivative of functions, namely the intermediate value property of derivative. We will 
extend this result to the multivalued case.

Theorem 5. Suppose $F: I \rightarrow \mathbb{R}$ is a multivalued function with closed, bounded and convex values. If $F$ is a derivative, then $F$ has the intermediate value property.

Proof. Assume the contrary. Then there exist two distinct points $x_{1}, x_{2} \in I$, say $x_{1}<x_{2}$, and a point $y_{1} \in F\left(x_{1}\right)$ such that for any $y \in F\left(x_{2}\right)$ there exists a number $\alpha$ with $\alpha \in\left(y_{1} \wedge y, y_{1} \vee y\right) \backslash F\left(\left(x_{1}, x_{2}\right)\right)$. Obviously $y_{1} \notin F\left(x_{2}\right)$. Let $y_{2}=\inf F\left(x_{2}\right)$. We have either $y_{1}<y_{2}$ or $y_{1}>y_{2}$. Let us suppose that $y_{1}<y_{2}$ and

$$
\alpha \in\left(y_{1}, y_{2}\right) \backslash F\left(\left(x_{1}, x_{2}\right)\right) .
$$

On the other hand there is by hypothesis a differentiable multivalued function $\Phi: I \rightarrow \mathbb{R}$ such that $F(x)=D \Phi(x)$ for each $x \in I$. It follows from Theorem 3 that $\Phi$ is $h$-continuous. Assume $\Phi(x)=[i(x), s(x)]$ (see (8)). Then the functions $i$ and $s$ are continuous (see (10)).

Let $K=\bigcup\left\{S i^{\prime}(x): x \in\left(x_{1}, x_{2}\right) \cap \Delta_{S}(i)\right\}$ and let $L=\bigcup\left\{S s^{\prime}(x): x \in\right.$ $\left.\left(x_{1}, x_{2}\right) \cap \Delta_{S}(s)\right\}$, where $\Delta_{S}(i)$ and $\Delta_{S}(s)$ denote the sets of points at which $i$ and $s$, respectively, are semiderivable. By Theorem $\mathrm{F}$ both sets $K$ and $L$ are connected.

Let us remark, that

$$
\begin{gathered}
\text { If } x \in\left[x_{1}, x_{2}\right] \text { and } z \in\left\{D_{+} i(x), D_{-} i(x), D^{+} i(x), D^{-} i(x)\right\}, \\
\text { then } z \text { is a limit point of } K .
\end{gathered}
$$

Similarly, if $x \in\left[x_{1}, x_{2}\right]$ and $z \in\left\{D_{+} s(x), D_{-} s(x), D^{+} s(x), D^{-} s(x)\right\}$,

then $z$ is a limit point of $L$.

In fact, without loss of generality we can assume $z=D^{+} i(x)$. Thus there is a sequence $\left(x_{n}\right)$ which converges to $x$ from the right such that

$$
\lim _{n \rightarrow \infty} Q i\left(x, x_{n}\right)=z .
$$

We conclude from Theorem $\mathrm{E}$ that for each $n \in \mathbb{N}$ there exists $y_{n} \in\left(x, x_{n}\right)$ such that $i$ is semiderivable at $y_{n}$ and

$$
Q i\left(x, x_{n}\right) \in S i^{\prime}\left(y_{n}\right) \in K \text {. }
$$

By (14) and (15) we have (13).

Suppose $F\left(x_{1}\right)=[p, q]$ and $F\left(x_{2}\right)=\left[y_{2}, r\right]$. Then according to (12) we have

$$
p \leq y_{1}<\alpha<y_{2} \leq r .
$$


Let us suppose $p \in\left\{D_{+} i\left(x_{1}\right), D^{+} i\left(x_{1}\right)\right\}$. One of the points $y_{2}$ or $r$ belongs to the set $\left\{D_{-} i\left(x_{2}\right), D^{-} i\left(x_{2}\right)\right\}$. Suppose $y_{2}$. Then according to (13) $p$ and $y_{2}$ are the limit points of $K$. The set $K$ is connected. Therefore $\left(p, y_{2}\right) \subset K$ and by (16) $\alpha \in K$. Similarly if $p \in\left\{D_{+} s\left(x_{1}\right), D^{+} s\left(x_{1}\right)\right\}$, then $\alpha \in L$. Therefore $\alpha \in K \cup L$. Let us note that $K \cup L \subset F\left(\left(x_{1}, x_{2}\right)\right)$. But this contradicts (12). We obtain a similar conclusion when $y_{1}>y_{2}$.

\section{The $\mathcal{D}_{*}$ Property of Approximately Continuous Multi- valued Functions}

Let $(Y, \mathcal{T}(Y))$ be a topological space, let $F: I \rightarrow Y$ be a multivalued function and $x_{0} \in I$.

Definition 4. $F$ is called approximately upper (resp. lower) semicontinuous at the point $x_{0}$ if there exists a set $A \in \mathcal{L}(\mathbb{R})$ such that density $D\left(x_{0}, A\right)=1$ and the restriction $\left.F\right|_{A}$ is $h$-upper (resp. $h$-lower) semicontinuous at $x_{0}$.

If $F$ is simultaneously approximately upper and lower semicontinuous at $x_{0}$, then it is called approximately continuous at $x_{0}$.

The following assertion is known (see [Kw, Prop. 1]).

Theorem G. If a multivalued function $F: I \rightarrow Y$ is almost everywhere approximately upper (resp. lower) semicontinuous, then it is upper (resp. lower) measurable.

From now on, let $Y$ be a reflexive Banach space. Let $T \in \mathcal{L}(\mathbb{R})$ and $F: T \rightarrow Y$ be a multivalued function which is lower measurable and bounded (in the sense that all its values are contained in a fixed totally bounded set $K)$ with $F(x) \in \mathcal{C}_{o b}(Y)$ for $x \in T$.

Let $E \subset T$ be a bounded Lebesgue measurable set. We define an integral of $F$ on $E$ as follows. (Compare [Hk, p.218] for the case $Y=\mathbb{R}^{n}$.)

If $F$ takes only a finite number of values $B_{1}, B_{2}, \ldots, B_{n}$ : i.e.,

$$
F(x)=\sum_{i=1}^{n} \chi_{D_{i}}(x) \cdot B_{i},
$$

where $D_{i}=\left\{x \in T: F(x)=B_{i}\right\}$ for $i=1,2, \ldots, n$, then we put

$$
\int_{E} F(x) d x=\sum_{i=1}^{n}\left|E \cap D_{i}\right| \cdot B_{i} \in \mathcal{C}_{o b}(Y) .
$$


Using Theorem B (i), we find that

if $A, B \in \mathcal{L}(\mathbb{R})$ are non-overlapping, bounded sets such that

$$
E=A \cup B, \text { then } \int_{E} F(x) d x=\int_{A} F(x) d x+\int_{B} F(x) d x .
$$

If $F$ and $G$ take a finite number of values, then using Theorem B (iii) one obtains

$$
d_{H}\left(\int_{E} F(x) d x, \int_{E} G(x) d x\right) \leq \int_{E} d_{H}(F(x), G(x)) d x .
$$

For a general measurable bounded multivalued function the definition of integral is based on the following lemma.

Lemma 1. Let a totally bounded convex set $K \subset Y$ and a number $\delta>0$ be given. Then there exists a finite family $\mathcal{F}_{\delta}$ of nonempty, closed, bounded and convex subsets of $Y$ such that if $D \in \mathcal{C}_{o b}(K)$, then there exists a smallest set $B \in \mathcal{F}_{\delta}$ such that $D \subset B \subset B(D, \delta)$.

Proof. Let $K \subset Y$ be totally bounded. Then $\mathcal{C}_{b}(K)$ is totally bounded in $\mathcal{C}_{b}(Y)$ (see [Kt, theorem 2, p. 113]), and then any sequence of elements in $\mathcal{C}_{b}(K)$ contains a Cauchy subsequence (see [Ha, theorem II, p.108]). Therefore any sequence of elements in $\mathcal{C}_{o b}(K) \subset \mathcal{C}_{b}(K)$ contains a Cauchy subsequence whose limit is in $\mathcal{C}_{o b}(K)$ by Theorem $\mathrm{C}$ ( $K$ is complete); i.e., $\mathcal{C}_{o b}(K)$ is compact. Let $\mathcal{K}$ be a finite subfamily of $\mathcal{C}_{o b}(K)$ such that for every $C \in \mathcal{C}_{o b}(K)$ there is $D \in \mathcal{K}$ such that $d_{H}(C, D)<\frac{\delta}{2}$. Then $C \subset \operatorname{cl} B\left(D, \frac{\delta}{2}\right)$. Let $\mathcal{L}=\left\{\operatorname{cl} B\left(D, \frac{\delta}{2}\right):\right.$ $D \in \mathcal{K}\}$. Then the family of all nonempty intersections of sets from $\mathcal{L}$ is the required family $\mathcal{F}_{\delta}$.

Now, take the $K$ in the lemma to be the totally bounded set containing all the values of $F$. Suppose $x \in T$. Let $\mathcal{F}_{\delta}$ be the family corresponding to $\delta>0$, and let $F_{\delta}(x)$ be the smallest member of $\mathcal{F}_{\delta}$ containing $F(x)$. Then $d_{H}\left(F(x), F_{\delta}(x)\right)<\delta$, multivalued function $F_{\delta}: T \rightarrow Y$ takes only finite number of values and by (18) and Theorem $\mathrm{C} \lim _{\delta \rightarrow 0} \int_{E} F_{\delta}(x) d x$ exists in $\mathcal{C}_{o b}(Y)$ and we take this limit to be the integral of multivalued function $F$ on $E$; i.e.

$$
\int_{E} F(x) d x=\lim _{\delta \rightarrow 0} \int_{E} F_{\delta}(x) d x
$$

By a passage to a limit in (17) (resp. in (18)) we obtain the corresponding equality (resp. inequality) for bounded, measurable multivalued functions. 
Theorem 6. If a bounded multivalued function $F:[a, b] \rightarrow Y$ with closed and convex values is approximately continuous, then it is a derivative.

Proof. Suppose that $F$ is approximately continuous. By Theorem G and Theorem $\mathrm{D}, F$ is lower measurable, and since it is also bounded, the integral of $F$ exists on any measurable subset of $[a, b]$.

Define a multivalued function $\Phi:[a, b] \rightarrow Y$ by $\Phi(x)=\int_{a}^{x} F(t) d t$. We will show that $F$ is the derivative of $\Phi$.

Let $x_{0} \in[a, b]$. Since $F$ is approximately continuous at $x_{0}$, there exists a measurable set $A \subset[a, b]$, such that $D\left(x_{0}, A\right)=1$ and $\left.F\right|_{A}$ is $h$-continuous at $x_{0}$. Suppose $\Delta x>0$ and $x_{0}+\Delta x \in[a, b]$. Then by (19)

$$
\Phi\left(x_{0}+\Delta x\right)=\Phi\left(x_{0}\right)+\int_{x_{0}}^{x_{0}+\Delta x} F(x) d x
$$

and by this

$$
\Phi\left(x_{0}+\Delta x\right) \ominus \Phi\left(x_{0}\right)=\int_{x_{0}}^{x_{0}+\Delta x} F(x) d x .
$$

Note, that by (19) we have

$$
\begin{aligned}
d_{H} & \left(\frac{\Phi\left(x_{0}+\Delta x\right) \ominus \Phi\left(x_{0}\right)}{\Delta x}, F\left(x_{0}\right)\right)=d_{H}\left(\frac{1}{\Delta x} \int_{x_{0}}^{x_{0}+\Delta x} F(x) d x, F\left(x_{0}\right)\right) \\
= & d_{H}\left(\frac{1}{\Delta x} \int_{x_{0}}^{x_{0}+\Delta x} F(x) d x, \frac{1}{\Delta x} \int_{x_{0}}^{x_{0}+\Delta x} F\left(x_{0}\right) d x\right) \\
\leq & \frac{1}{\Delta x} \int_{x_{0}}^{x_{0}+\Delta x} d_{H}\left(F(x), F\left(x_{0}\right)\right) d x \\
= & \frac{1}{\Delta x} \int_{\left[x_{0}, x_{0}+\Delta x\right] \cap A} d_{H}\left(F(x), F\left(x_{0}\right)\right) d x \\
& +\frac{1}{\Delta x} \int_{\left[x_{0}, x_{0}+\Delta x\right] \backslash A} d_{H}\left(F(x), F\left(x_{0}\right)\right) d x .
\end{aligned}
$$

$F$ is bounded. Let $K$ be the fixed totally bounded set which includes all the values of $F$. As $\Delta x$ tends to 0 , the first term of the above expression converges to 0 since $F$ is $h$-continuous on $A$, and the second is majorized by $\frac{1}{\Delta x}\left|\left[x_{0}, x_{0}+\Delta x\right] \backslash A\right| 2 \mid\|K\|$, which converges to 0 , since $D\left(x_{0},[a, b] \backslash A\right)=0$. This, together with a similar calculation for $\Delta x<0$ and $x_{0}+\Delta x \in[a, b]$, yields

$$
d_{H}\left(\Phi\left(x_{0}\right) \ominus \Phi\left(x_{0}+\frac{\Delta}{x}\right) \Delta x, F\left(x_{0}\right)\right) \leq \varepsilon,
$$


and by this it follows that $D \Phi\left(x_{0}\right)=F\left(x_{0}\right)$. Hence that $F$ is a derivative and the proof of Theorem 5 is finished.

By Theorem 5 and Theorem 6 we have

Conclusion 1. If a bounded multivalued function $F: I \rightarrow \mathbb{R}$ with closed and convex values is approximately continuous, then it has the intermediate value property.

Acknowledgements The author is greatly indebted to Tim Traynor for many stimulating conversations during the preparation of this paper.

\section{References}

[CK] J. Czarnowska, G. Kwiecińska, On the Darboux property of multivalued functions, Demonstr. Math. XXV (1992), No.1-2, 192-199.

[EL] J. Ewert, J. S. Lipiński, On the continuity of Darboux multifunctions, Real Anal. Ex. 13, No 1 (1987-88), 122- 125.

[G1] K. M. Garg, A new notion of derivative, Real Anal. Ex., Vol. 7 (19811982), 65-84.

[G2] K. M. Garg, Theory of Differentiation, A Wiley-Interscience Publication, vol. 24, 1998.

[Ha] F. Hausdorff, Mengenlehre, Berlin and Lipzig, de Gruyter, 2d edition, 1927.

[Hk] M. Hukuhara, Integration des applications measurables dont la valuer est un compact convexe, Funkcialaj Ekvacioj 10 (1967), 205-223.

[Kt] K. Kuratowski, Topologie I, Monografie Matematyczne,Warsaw, 1958.

[Kw] G. Kwiecińska, Approximately continuous multivalued functions in abstract spaces, Proceedings of the Eight Prague Topological Symposium (1996), 191-197 (electronic), Topol. Atlas, North Bay, ON 1997.

[KŚ] G. Kwiecińska, Ślęzak W.,On complete measurability of multifunctions defined on product spaces, Acta Math. Univ. Comenianae, vol. LXVI, 2 (1997), 293-305.

[Lc] A. Lechicki, Continuous and measurable multifunctions, Commentationes Mathematicae, vol.XXXI, 1, (1980), 141-156. 
[Pc] G. B. Price, The theory of integration, Trans. Amer. Math. Soc., 47, 1940, 1-50.

[Rd] H. Radström, An embedding theorem for spaces of convex sets, Proc. Amer. Math. Soc. 3 (1952), 165-169. 